\title{
Paradigm shift in medical ultrasonography
}

\author{
Shoichi Matsutani
}

(C) The Japan Society of Ultrasonics in Medicine 2012

Novel innovations in science and technology are being developed every minute somewhere in the world. Although these developments may make our lives and work conditions better than previously, every innovation does not necessarily bring us happiness. In our field of medical ultrasonography, progress over the past two decades, including contrast-enhancement technology and 3D or 4D images, has changed our daily clinical practice tremendously. From my personal point of view, however, one novel development in ultrasound technology that occurred almost 40 years ago was quite important because it gave me a chance to write this editorial by prompting me to study and practice medical ultrasound. The technical development to which I am referring is the great progress in ultrasound technology associated with electronic scans, etc., which led to the present real-time grayscale ultrasonography. It may seem a little bit naïve to the young members of our Society. For myself, however, who just started medical training as a young physician, the impact was great. This feeling must be the same for many grayhaired members who were young at that time and more elderly members who may have been young at heart at that time, resembling a paradigm shift in 20th-century medicine. This progress in medical ultrasound also changed the life of one special person from that time who passed away in January 2012 (Masao Ohto, M.D., 1929-2012: Professor Emeritus, Chiba University; President of JSUM Annual Meeting 1993). Before then, his major concerns were the development of the very fine needle and its clinical applications for the diagnosis of hepatobiliary and pancreatic diseases (Cholangiography and Pancreatography, Igaku Shoin, Tokyo/New York, 1978). The needle he developed, known as the Chiba needle, is now widely used in various clinical settings for hepatobiliary and pancreatic diseases around the world. Since the middle of the 1970s, when new technologies were being applied in ultrasound medicine, he devoted his life to the development of early diagnosis and nonsurgical treatment of liver cancer, which is still of grave concern worldwide. An ultrasonically guided alcohol injection to liver cancer, which he developed, was a sophisticated collaboration of his fine needle with ultrasound medicine as a first arrow aimed at the newly developing field of nonsurgical and less-invasive local treatment for liver cancer at that time (Acta Hepatologica Japonica 1983;24:920).

Therapeutic uses of medical ultrasound have been our major concern since ultrasound was employed in medicine. We will soon have real therapeutic applications of ultrasound such as the use of high-intensity focused ultrasound for the treatment of tumors or caliculi and the use of ultrasound for thrombolysis. Ultrasound medicine undoubtedly has the potential to offer less-invasive or noninvasive treatment for various diseases. In the near future, we may be able to diagnosis and treat many diseases using a system whereby the diagnosis is made with ultrasound followed by treatment with ultrasound on the same day. Although further progress in the science and technology of medical ultrasound is necessary to realize that point, clinical practice employing only ultrasound will be our ultimate goal as the next paradigm shift in 21st-century medicine.

S. Matsutani $(\bowtie)$

Chiba Prefectural University of Health Science, Chiba, Japan

e-mail: shoichi.matsutani@cpuhs.ac.jp 